06

\title{
Условия получения высокомодульных нанокомпозитов полимер/углеродные нанотрубки
}

\author{
() Г.В. Козлов, И.В. Долбин \\ Кабардино-Балкарский государственный университет им. Х.М. Бербекова, \\ 360004 Нальчик, Россия \\ e-mail: i_dolbin@mail.ru
}

Поступило в Редакцию 8 апреля 2020 г.

В окончательной редакции 20 сентября 2020 г.

Принято к публикации 21 сентября 2020 г.

Рассмотрены физические основы реализации высокомодульных и высокопрочных нанокомпозитов полимер/углеродные нанотрубки с механическими характеристиками, сравнимыми с таковыми для стали. Определены два основных фактора, позволяющих создание таких нанокомпозитов - структура нанонаполнителя в полимерной матрице и достаточно высокое содержание нанонаполнителя. Фрактальная размерность указанной структуры должна быть близкой к размерности окружающего евклидова пространства, т.е. трем. Показано, что дополнительная вытяжка нанокомпозита дает два положительных эффекта: снижение волнистости углеродных нанотрубок и повышение модуля упругости полимерной матрицы вследствие ориентации ее макромолекул.

Ключевые слова: высокомодульный нанокомпозит, углеродные нанотрубки, модуль упругости, структура, фрактальный анализ.

DOI: $10.21883 /$ JTF.2021.03.50521.121-20

\section{Введение}

С момента своего появления в 1991 г. углеродные нанотрубки (УНТ) считались очень перспективным нанонаполнителем для создания полимерных нанокомпозитов в силу своих базовых характеристик: высокого продольного модуля упругости, сильной анизотропии и большой удельной поверхности [1]. Однако на практике потенциально высокие показатели нанокомпозитов полимер/УНТ реализуются редко, что в основном связано с рядом технологических факторов, основным из которых является агрегация исходных нанотрубок и их небольшое $(<5$ mass. \%) содержание. Кроме того, сильное отрицательное влияние на свойства конечных нанокомпозитов оказывает волнистость (непрямолинейность) УНТ [2]. Это обстоятельство предопределило разработку ряда технологических методов, позволяющих получение хорошо ориентированных волокон или листов УНТ и, в конечном итоге, высокомодульных и высокопрочных нанокомпозитов полимер/УНТ [2-4]. Так, авторы [2] получили нанокомпозиты бисмалеимид/УНТ (БМИ/УНТ) с объемным содержанием нанонаполнителя $\varphi_{n}=0.46$, которые имеют модуль упругости $293 \mathrm{GPa}$ и прочность $3.8 \mathrm{GPa}$, что сравнимо с показателями промышленных металлов, в частности, стали. Если учесть низкую плотность полученных таким образом нанокомпозитов $\left(1250 \mathrm{~kg} / \mathrm{m}^{3}\right)$ по сравнению с металлами, то становится очевидным потенциал их применения в аэрокосмической промышленности, судо- и автомобилестроении и т.п.

Однако эти несомненные технологические достижения не получили должного теоретического обоснования, необходимого для моделирования, прогнозирования и определения предельных характеристик рассматриваемых нанокомпозитов. Если такие обоснования и приводились, то только на качественном уровне. Так, авторы [4] предложили пять фундаментальных критериев, реализация которых позволяет получить нанокомпозиты полимер/УНТ высокого качества:

1) высокая степень анизотропии нанонаполнителя;

2) ориентация УНТ;

3) большое содержание нанонаполнителя;

4) высокая межфазная площадь (гомогенная дисперсия нанонаполнителя в полимерной матрице);

5) эффективный перенос механического напряжения между компонентами нанокомпозита.

Более детальный анализ этих положений показывает, что не все они являются независимыми. Например, между критериями 4 и 5 имеется очевидная взаимосвязь [5]. Кроме того, этот анализ не включает аналитического физического аппарата, позволяющего получить количественное соотношение указанных показателей. Поэтому целью настоящей работы является теоретический анализ структуры и свойств нанокомпозитов БМИ/УНТ, полученных специальными методами изготовления и имеющих исключительно высокие механические характеристики, в рамках общей для всех классов полимерных нанокомпозитов фрактальной модели [6].

\section{1. Методика эксперимента}

В настоящей работе выполнен анализ результатов, полученных авторами [2]. Основным фактором используемой технологии получения нанокомпозитов БМИ/УНТ 
является распрямление обладающих определенной степенью волнистости УНТ перед внедрением их в полимерную матрицу. Для этой цели использованы ориентированные листы УНТ, полученных методом химического осаждения паров. Они состояли из многослойных (4-6 слоев) УНТ диаметром 7-9 nm. В процессе изготовления листа УНТ он перемещался горизонтально и вытягивался при деформации вытяжки $\lambda$, определяемой следующим образом [2]:

$$
\lambda=\frac{L-L_{0}}{L_{0}} \cdot 100[\%],
$$

где $L$ и $L_{0}$ - длина листа УНТ после и до вытяжки.

Затем вытянутый лист УНТ наматывался на вращающуюся цилиндрическую катушку из политетрафторэтилена.

В качестве матричного полимера использован бисмалеимид (БМИ) с удлиненным имидом высокого качества производства фирмы Designer Molecules Inc. (США). Раствор БМИ в толуоле с концентрацией $1.0 \mathrm{~g} / 1$ наносился распылителем от слоя к слою по мере намотки листа УНТ на катушку [2].

Испытания на растяжение выполнены на образцах в форме двухсторонней лопатки длиной $40 \mathrm{~mm}$, шириной $20 \mathrm{~mm}$ и толщиной $0.02-0.04 \mathrm{~mm}$. В этих испытаниях использована базовая длина образца $20 \mathrm{~mm}$ при скорости смещения ползуна $1.0 \mathrm{~mm} / \mathrm{min}$. Для испытаний применяли прибор Micro Autograph MST-I производства фирмы Shimadzu Co. (Япония) с ячейкой нагрузки $100 \mathrm{~N}$ [2].

\section{2. Результаты и их обсуждение}

В настоящее время известно $[7,8]$, что свойства полимерных нанокомпозитов определяются структурой нанонаполнителя в полимерной матрице. Авторы [6] предложили следующее уравнение для описания механических свойств полимерных нанокомпозитов:

$$
\frac{E_{n}}{E_{m}}=1+17 D_{f}^{2} \varphi_{n},
$$

где $E_{n}$ и $E_{m}-$ модули упругости нанокомпозита и матричного полимера соответственно (отношение $E_{n} / E_{m}$ принято называть степенью усиления нанокомпозита), $D_{f}$ - фрактальная размерность нанонаполнителя в полимерной матрице, характеризующая его структуру, $\varphi_{n}$ - объемное содержание нанонаполнителя.

Для рассматриваемых нанокомпозитов БМИ/УНТ $E_{m}=4 \mathrm{GPa}, \varphi_{n}=0.46$ [2]. Уравнение (2) позволяет оценить величину $D_{f}$ по известным значениям $E_{n} / E_{m}$ и $\varphi_{n}$. Авторы [2] использовали три типа нанокомпозитов БМИ/УНТ, для которых деформация вытяжки листов УНТ составляла 0,10 и $12 \%$ (см. уравнение (1)). В таблице приведены величины $E_{n}$ и $D_{f}$ для трех указанных величин $\lambda$. Как следует из этих данных, распрямление УНТ, обусловленное их вытяжкой, приводит к увеличению как $E_{n}$, так и $D_{f}$, причем при $\lambda=12 \%$ величина
Структурные и механические характеристики нанокомпозитов БМИ/УНТ

\begin{tabular}{l|c|c|c|c|c|c}
\hline$\lambda, \%$ & $\varphi_{n}$ & $E_{n}, \mathrm{GPa}$ & $D_{f}$ & $E_{n a n}, \mathrm{GPa}$ & $D_{f}^{\mathrm{CNT}}$ & $R_{\mathrm{CNT}}, \mu \mathrm{m}$ \\
\hline 0 & 0.46 & 120 & 1.93 & 253 & 1.68 & 6.1 \\
10 & 0.46 & 220 & 2.63 & 470 & 2.88 & 2191 \\
12 & 0.46 & 293 & 3.0 & 612 & 2.93 & 225560
\end{tabular}

$D_{f}$ достигает своего предельного значения $D_{f}=d=3$, где $d$ - размерность евклидова пространства, в котором рассматривается фрактал. Это означает, что дальнейшая вытяжка с $\lambda>12 \%$ не имеет смысла, поскольку она не может привести к повышению $D_{f}$ и, следовательно, $E_{n} / E_{m}$, но может быть причиной разрушения отдельных нанотрубок в листе УНТ (рис. 1).

Модуль упругости нанонаполнителя в полимерной матрице $E_{\text {nan }}$ можно определить согласно следующему уравнению [6]:

$$
E_{\text {nan }}=17 D_{f}^{2} E_{m}
$$

Рассчитанные согласно этому уравнению значения $E_{\text {nan }}$, также приведенные в таблице, позволяют сделать два вывода. Во-первых, при $\lambda=12 \%$ или $D_{f}=3$ величина $E_{n a n}=612 \mathrm{GPa}$, что близко к номинальному модулю индивидуальной углеродной нанотрубки, изготовленной методом химического осаждения паров, равному $\sim 500 \mathrm{GPa}[9]$.

Следует отметить, что величина номинального модуля упругости $E_{\mathrm{CNT}}$ для нанотрубок, полученных методом химического осаждения паров, колеблется в очень широком диапазоне и зависит от используемых реагентов, геометрии нанотрубок, их дефектности и т.П. Кроме того, измерение $E_{\mathrm{CNT}}$ для каждого конкретного случая - процедура сложная, трудоемкая и требующая специального оборудования. Поэтому, как правило, для целей сравнения пользуются литературными данными. Мы поступили так же. В данном конкретном случае важно приблизительное соответствие величин $E_{\text {nan }}$ и $E_{\mathrm{CNT}}$, поскольку значения $E_{\mathrm{CNT}}$ в работе [2] не приведены и нет гарантии, что они, допустим, не лежат в диапазоне 600-700 GPa. Тем не менее случаи превышения величинами эффективного модуля упругости $E_{n a n}$ номинального модуля упругости $E_{\mathrm{CNT}}$ встречались и ранее $(1.5$ и $1.0 \mathrm{GPa}$ соответственно) [9], но объяснить этот эффект авторы не смогли. Частичный ответ на этот вопрос дает уравнение (3). Если каким-либо способом можно получить матричный полимер с модулем упругости $E_{m}=10 \mathrm{GPa}$, то при $D_{f}=3$ мы получим $E_{\text {nan }}=1530 \mathrm{GPa}$, что в 1.5 раза больше наиболее высоких цитируемых в литературе номинальных значений $E_{\mathrm{CNT}}=1000 \mathrm{GPa}$. В принципе такая схема возможна, например, ориентацией полимерной матрицы или созданием гибридных нанокомпозитов.

Сказанное выше означает, что потенциально высокий модуль упругости отдельной УНТ полностью реализуется в рассматриваемых нанокомпозитах при условии 


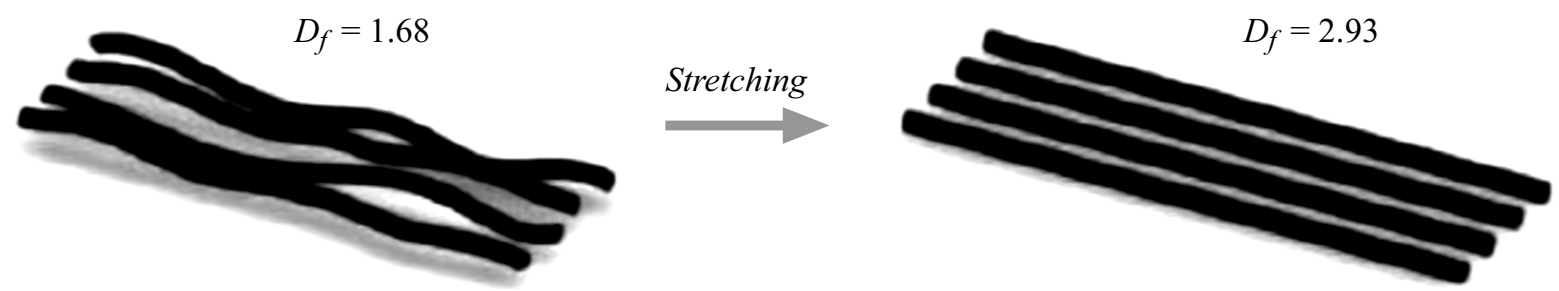

Рис. 1. Схематическая иллюстрация концепции распрямления УНТ [2].

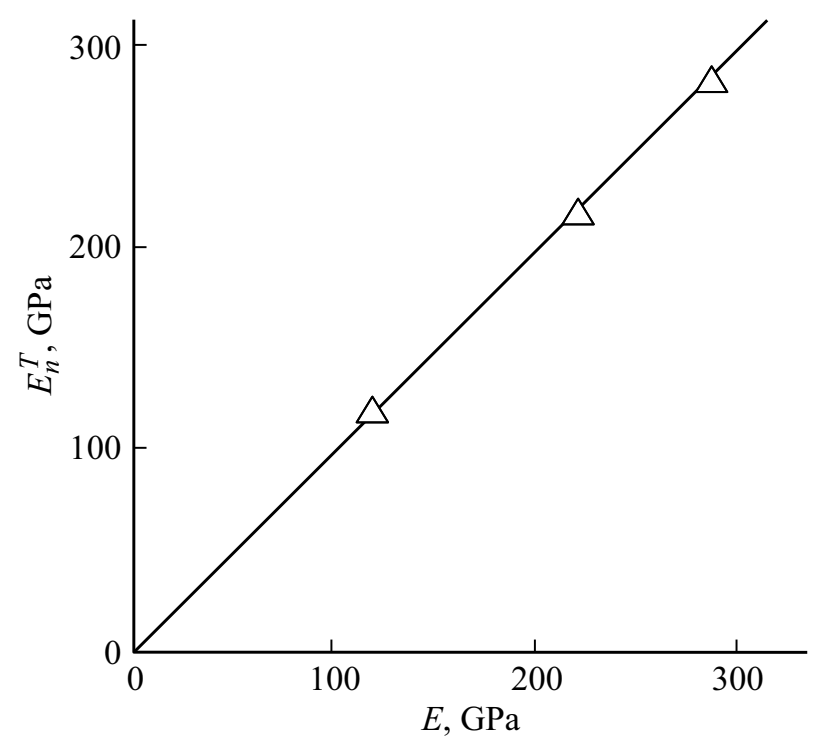

Рис. 2. Сравнение полученных экспериментально $E_{n}$ и рассчитанных, согласно правилу смесей (уравнение (5)) $E_{n}^{T}$, величин модуля упругости для нанокомпозитов БМИ/УНТ.

$\lambda=12 \%$. Кроме того, уравнение (3) демонстрирует зависимость модуля упругости нанонаполнителя $E_{\text {nan }}$ от жесткости окружающей среды, т.е. полимерной матрицы. Это правило является общим для полимерных нанокомпозитов всех классов и в случае стеклообразной полимерной матрицы описывается следующим простым соотношением [10]:

$$
E_{\text {nan }}=22 E_{m}^{2}
$$

Оценка, согласно уравнению (4), дает $E_{\text {nan }}=352 \mathrm{GPa}$, что достаточно хорошо согласуется с оценкой по уравнению (3) (см. таблицу).

Проверку корректности рассчитанных, согласно уравнению (3), величин $E_{\text {nan }}$ можно выполнить с помощью правила смесей в его простейшей изначальной формe [10]:

$$
E_{n}^{T}=E_{n a n} \varphi_{n}+E_{m}\left(1-\varphi_{n}\right) .
$$

Результаты расчета теоретических значений модуля упругости нанокомпозитов БМИ/УНТ приведены на рис. 2, откуда следует их превосходное соответствие экспериментальным данным. Такое соответствие демонстрирует, что вопреки многочисленным сомнениям в корректности применения правила смесей для описания свойств нанокомпозитов [11] это правило справедливо, если в нем используются реальные, а не номинальные значения модуля упругости нанонаполнителя $E_{n a n}$. Далее рассмотрим поведение отдельных углеродных нанотрубок в их агрегатах для рассматриваемых нанокомпозитов. Предполагая, что кластер УНТ с размерностью $D_{f}$ формируется взаимодействием отдельных нанотрубок с полимерной матрицей, для определения размерности этих нанотрубок $D_{f}^{\mathrm{CNT}}$ можно использовать следующую формулу [12]:

$$
D_{f}=\frac{3\left(2 D_{f}^{m}-D_{f}^{\mathrm{CNT}}\right)}{3+2\left(D_{f}^{m}-D_{f}^{\mathrm{CNT}}\right)},
$$

где $D_{f}^{m}$ - размерность макромолекулярного клубка матричного полимера, принятая для БМИ равной 1.87 [13].

Уравнение (6) было получено в рамках аппроксимации Флори применительно к механизму агрегации кластер-кластер [12]. Поскольку в рассматриваемом случае речь идет о взаимодействии кластеров (макромолекулярных клубков полимерной матрицы и кластеров УНТ), такая модель применима для описания результата их взаимодействия.

Величины $D_{f}^{\mathrm{CNT}}$ приведены в таблице, откуда следует их быстрый рост по мере увеличения деформации вытяжки $\lambda$. Волнистость УНТ можно охарактеризовать их радиусом кривизны $R_{\mathrm{CNT}}$, определяемым согласно уравнению [14]:

$$
R_{\mathrm{CNT}}=3.4\left(\varphi_{n}\right)^{-1 /\left(d-D_{f}^{\mathrm{CNT}}\right)},
$$

где $R_{\mathrm{CNT}}$ дается в $\mu \mathrm{m}$.

Приведенные в таблице величины RCNT показали очень быстрый рост по мере увеличения $\lambda$, что означает их сильное распрямление, и при $\lambda=10$ и 12\% УНТ в агрегате практически прямолинейны, что подтверждает вывод, сделанный по этому вопросу в работе [2].

\section{Заключение}

Таким образом, в настоящей работе предложена структурная (фрактальная) модель, позволяющая корректно описать степень усиления высокомодульных нанокомпозитов полимер/УНТ. Эта модель универсальна 
для основных классов полимерных нанокомпозитов и предполагает два основных фактора, определяющих степень усиления указанных наноматериалов: структурное состояние нанонаполнителя в полимерной матрице, характеризуемое его фрактальной размерностью, и содержание нанонаполнителя. Модуль упругости агрегатов нанонаполнителя зависит также от жесткости окружающей их среды, т.е. модуля упругости полимерной матрицы. Правило смесей в его простейшей изначальной форме дает корректное описание степени усиления нанокомпозитов при условии использования реальных, а не номинальных величин входящих в него характеристик.

\section{Конфликт интересов}

Авторы заявляют, что у них нет конфликта интересов.

\section{Список литературы}

[1] M. Moniruzzaman, K.I. Winey. Macromolecules, 39 (16), 5194 (2006). DOI: 10.1021/ma060733p

[2] X. Wang, Z.Z. Yong, Q.W. Li, P.D. Bradford, W. Liu, D.S. Tucker, W. Cai, H. Wang, F.G. Yuan, Y.T. Zhu. Mater. Res. Lett., 1 (1), 19 (2013). DOI: 10.1080/21663831.2012.685586

[3] Q.F. Cheng, J.P. Wang, J.J. Wen, C.H. Liu, K.L. Jiang, Q.Q. Li, S.S. Fan. Carbon, 48 (2), 260 (2010). DOI: 10.1016/j.carbon.2009.09.014

[4] K. Kobashi, H. Nishino, T. Yamada, D.N. Futaba, M. Yumura, K. Hata. Carbon, 49 (23), 5090 (2011). DOI: 1016/j.carbon.2011.07.028

[5] Х.Ш. Яхьяева, Г.М. Магомедов, Г.В. Козлов. Структура и адгезионные явления в полимерных системах (Перо, M., 2016)

[6] Г.В. Козлов, П.Г. Ризванова, И.В. Долбин, Г.М. Магомедов. Известия вузов. Физика, 62 (1), 112 (2019). [G.V. Kozlov, P.G. Rizvanova, I.V. Dolbin, G.M. Magomedov. Rus. Phys. J., 62 (1), 127 (2019). DOI: 10.1007/s11182-019-01692-1]

[7] D.W. Schaefer, J. Zhao, H. Dowty, M. Alexander, E.B. Orler. Soft Matter, 4 (10), 2071 (2008). DOI: 10.1039/b805314f

[8] Л.Б. Атлуханова, Г.В. Козлов, И.В. Долбин. Материаловедение, 7, 19 (2019). DOI: $10.31044 / 1684-579 x-2019-0-7-19-22$

[9] D. Blond, V. Barron, M. Ruether, K.P. Ryan, V. Nicolosi, W.J. Blau, J.N. Coleman. Adv. Funct. Mater., 16 (15), 1608 (2006). DOI: 10.1002/adfm.200500855

[10] Г.В. Козлов, И.В. Долбин. G.V. Нано- и микросистемная техника, 20 (8), 471 (2018). DOI: $10.17587 / \mathrm{nmst} .20 .466-474$

[11] H. Miyagawa, L.T. Drzal. Polymer, 45 (21), 5163 (2004). DOI: 10.1016/j.polymer.2004.05.036

[12] H.G.E. Hentschel, J.M. Deutch. Phys. Rev. A, 29 (3), 1609 (1984). DOI: 10.1103/PhysRevA.29.1609

[13] G.V. Kozlov, I.V. Dolbin, G.E. Zaikov. The Fractal Physical Chemistry of Polymer Solutions and Melts (Apple Academic Press, Toronto, New Jersey, 2014).

[14] А.К. Микитаев, Г.В. Козлов. ДАН, 462 (1), 41 (2015). [A.K. Mikitaev, G.V. Kozlov. Dokl. Phys., 60 (5), 203 (2015). DOI: $10.1134 / \mathrm{S} 102833581505002 \mathrm{X}]$ 